Article

\title{
Application of Endophytic Bacillus subtilis and Salicylic Acid to Improve Wheat Growth and Tolerance under Combined Drought and Fusarium Root Rot Stresses
}

\author{
Oksana Lastochkina ${ }^{1, *(\mathbb{D})}$, Darya Garshina ${ }^{2}$, Chulpan Allagulova ${ }^{1}$, Kristina Fedorova ${ }^{1}$, \\ Igor Koryakov ${ }^{1}$ and Anastasiya Vladimirova ${ }^{1}$ \\ 1 Institute of Biochemistry and Genetics-Subdivision of the Ufa Federal Research Centre of the Russian \\ Academy of Sciences, 450054 Ufa, Russia; allagulova-chulpan@rambler.ru (C.A.); \\ kristina-iva@yandex.ru (K.F.); koryakov_igor@mail.ru (I.K.); vladimirovaw@bk.ru (A.V.) \\ 2 Bashkir Research Institute of Agriculture-Subdivision of the Ufa Federal Research Centre of the Russian \\ Academy of Sciences; 450059 Ufa, Russia; dariya.greatfire@mail.ru \\ * Correspondence: oksanaibg@gmail.com; Tel.: +7-963-901-80-46
}

Received: 15 July 2020; Accepted: 3 September 2020; Published: 7 September 2020

check for updates

\begin{abstract}
In nature, plants are constantly exposed to a varied abiotic and biotic stresses or their combinations, limiting the productivity of major crops, including wheat. Combinations of drought and soil-borne Fusarium-instigated diseases are the most common combinations of stresses, significantly reducing wheat yield around the world. Here, were analyzed the potential of application of endophytic bacteria Bacillus subtilis (strain 10-4) together with the natural signal molecule salicylic acid (SA) to improve growth and tolerance of Triticum aestivum L. (wheat) plants under combined drought and Fusarium culmorum-instigated root rot (FRR) stresses. It was revealed that pre-sowing treatment with B. subtilis 10-4, SA, and B. subtilis 10-4 + SA, both under normal and combined drought conditions, notably reduced (by $50-80 \%$ or more) the incidence of FRR development in wheat plants, with the most notable effect for $B$. subtilis 10-4 + SA (wherein disease symptoms were almost absent). Moreover, B. subtilis 10-4, SA, and especially B. subtilis 10-4 + SA increased plant growth (root and shoot length, fresh and dry biomass) under normal (up to 20-50\%), drought (up to 15-40\%), FRR (up to 15-30\%), and combined drought + FRR stresses (up to 20\%), with the maximum effect for B. subtilis 10-4 + SA. Additionally, B. subtilis 10-4, SA, and B. subtilis 10-4 + SA decreased stress (drought, FRR, and combined drought + FRR)-instigated lipid peroxidation and osmotic damages of plant cells. The findings indicate that endophytic bacteria B. subtilis 10-4 alone and in a mixture with SA may be used as an effective eco-friendly agent to improve wheat growth and tolerance under the influence of drought, FRR, and combinations of these stresses.
\end{abstract}

Keywords: combined stress; drought; Fusarium infection; beneficial microbes; endophytic Bacillus subtilis; salicylic acid; wheat; combined stress tolerance

\section{Introduction}

Wheat (Triticum aestivum L.) is the second major crop (after maize) that has great importance in ensuring food security around the globe [1]. Crop losses from abiotic stresses, diseases, and their combinations can reach up to $50-80 \%$, and are dangerous for the agricultural and food industries. In the field, the impact of drought and soil-borne diseases are the most common combinations of stresses that significantly reduce wheat growth and yield [1-4]. Drought and Fusarium root rot (FRR) of cereal is caused by phytopathogenic fungus Fusarium spp. (F. culmorum, F. graminearum, F. avenaceum, 
F. oxysporum, etc.) and its combinations, which are found in all regions of cereal cultivation $[1,3,4]$. The pathogen inoculum spreads from infected grain, crop residues, and colonized soil [5]. Plants become infected both during seed germination and during the growth period [3,4]. The pathogens penetrating the roots populate the upper part and xylem, affecting the roots, tillering nodes, and the base of the stems. Severe infection may result in seedling dieback, a decrease in the total and productive biomass, and the formation of dead and underdeveloped spikes with shriveled grain [3,4]. Dry and warm weather with insufficient or unstable soil moisture contributes to the development of the disease, with weakened plants with reduced cell turgor being the most susceptible to Fusarium infection [6,7]. Thus, drought can directly affect plant-fungi interactions and influence the occurrence and spread of pathogens [1,2,5-7]. Drought inhibits plant growth and nutrient absorption, and increases plants susceptibility to soil-borne pathogens $[1,6,7]$. Plant responses to combined abiotic-biotic stresses are complex and include numerous molecular, cellular, and physiological adaptations [2]. In particular, drought and FRR, like other stress-factors, promote oxidative and osmotic stress, water deficit, and stomatal closure in plants, resulting in the reduction of leaf sizes [4,7], thereby decreasing plant growth and productivity. The chemical approaches successfully applied over recent decades to increase plant resistance and productivity have adversely affected the environment, leading to land degradation; soil, water, and air pollution; and loss of biodiversity [1]. The negative impact of pesticides on the environment and human health leads to the need to reduce their use in food production and stimulates scientists around the world to show interest in developing novel eco-friendly and bio-safe strategies to reduce the global abiotic/biotic stresses that threatens agriculture [1].

Beneficial endophytic microorganisms capable of enhancing plant growth and inducing plant defense reactions against environmental stresses may be considered for application in improved crop management strategies [1]. Of special interest are the endophytic bacterium Bacillus subtilis, a natural component of soil microbiota that plays an important role in maintenance of plant and soil health under stress [8]. Additionally, B. subtilis is a microorganism that is generally recognized as safe (GRAS-generally recognized as safe) to use in the food industry and safe for human health and for the environment [1]. These make B. subtilis an attractive agent for the development of safe biological products to protect plants and increase their productivity $[1,8]$. By now, numerous studies have shown that $B$. subtilis promotes plant growth and induces resistance/tolerance throughout the host plants to a wide range of biotic (pathogens, pests) and abiotic stresses (drought, extreme temperatures, salinity, high lights, heavy metals, etc.), thereby leading to increased crop productivity [1]. B. subtilis is capable of inducing plant growth and anti-stress physiological programs through direct and indirect mechanisms, including the synthesis a broad spectrum of bioactive compounds with antibiotic activity, siderophores, chelators, enzymes, phytohormones, as well as regulating their levels in plants, fixing atmospheric nitrogen, improving macro-/micronutrient bioavailability, and inducing plant systemic resistance and tolerance [1,8,9]. Nevertheless, the knowledge of underlying mechanisms of their action is still unclear and requires further detailed investigation. Moreover, recent research has mainly focused on comprehension the mechanisms of B. subtilis action on responses of plants to either biotic or abiotic stresses separately $[1,9,10]$. However, plant responses to simultaneous stresses inevitably may result in a much more complex scenario, and knowledge on how B. subtilis can modulate plant-pathogen interactions under the adverse drought stress is limited. Unraveling the actual impact of $B$. subtilis on factors related to the yield of major crops such as wheat under combined stresses is also needed. Thus, the effectiveness of $B$. subtilis application to increase resistance to different stresses was shown at different plant objects, indicating that work on the development of biological products based on B. subtilis for plant protection against stresses is promising [1,9]. At present, the development of endophytic bacteria-based products is mainly hampered by the lack of knowledge of the underlying mechanisms of interactions between endophytic bacteria B. subtilis and wheat during biotic/abiotic stress. Moreover, it is difficult to select one microbial strain that simultaneously possesses a spectrum of activities against a number of pathogens and abiotic stresses. This is why there is an interest in application of $B$. subtilis together with other microbial strains and/or biological compounds, 
including natural and safe signaling molecules with pronounced anti-stress physiological activities, on plants such as salicylic acid (SA) and unraveling the mechanisms of their actions. SA is a recognized inducer of plants' systemic resistance against biotic and abiotic stress factors [11]. A large body of information accumulated up until today has indicated the involvement of SA in the regulation of defense reactions of different plants, including wheat, to phytopathogens and abiotic stresses [11,12]. However, the knowledge on the influence of endophytic B. subtilis, both alone and in a mixture with SA, on wheat growth and tolerance under the combined drought and FRR stresses are completely absent. In this regard, it is of great relevance to study the peculiar properties of wheat defense mechanisms to the combined effects of drought and FRR stresses upon B. subtilis and SA influences, which will unravel the mechanisms of wheat resistance formation under the influence of these growth regulators for their targeted use, as well as unleashing the genetic potential and increasing wheat productivity.

In this study, we analyzed the effect of seed priming by endophytic bacteria B. subtilis (strain 10-4) alone and together with SA on morpho-physiological traits of Triticum aestivum L. (wheat) plants under normal and combined drought and Fusarium culmorum-instigated root rot stress conditions.

\section{Materials and Methods}

\subsection{Bacterial Strain and Phytopathogenic Fungi}

Beneficial bacterial strain B. subtilis 10-4 was isolated from the arable soils (at the Bashkir Research Institute of Agriculture (BRIA) UFRC RAS (Ufa, Russia)) and has been described in our previous work [13]. In short, the bacteria B. subtilis 10-4 is Gram-positive, rod-shaped, aerobic, and motile. The colonies of $B$. subtilis 10-4 on potato glucose agar (PGA) are beige-white in color, round in shape with a wavy edge, having a smooth surface, and being rough in the center. On the basis of morphological characteristics and using a sequencing analysis of the variable regions of genes encoding 16S rRNA with a similarity of $99 \%$, the strain 10-4 was identified as B. subtilis [13]. In present study, the cells of $B$. subtilis $10-4$ were cultured on PGA medium ( $37^{\circ} \mathrm{C}$ over $3-4$ days) $[13,14]$. B. subtilis $10-4$ inoculum was obtained by preparation of strain $10-4$ suspension $\left(10^{8}\right.$ colony-forming units (CFU) $\left.\mathrm{mL}^{-1}\right)$ using $0.5 \mathrm{McF}$ arland Turbidity Standard and monitored by the optical density at $600 \mathrm{~nm}\left(\mathrm{OD}_{600}\right)$ (SmartSpec Plus spectrophotometer, Bio-Rad, Hercules, CA, USA). Thereafter, the bacterial suspension, containing $10^{8} \mathrm{CFU} \mathrm{mL}^{-1}$, was diluted with distilled water or/and salicylic acid (SA) solution $(0.05 \mathrm{mM})$ in distilled water to $10^{5} \mathrm{CFU} \mathrm{mL} \mathrm{mL}^{-1}$.

Fusarium culmorum (FRR causative agent) was provided by the Laboratory of Plant-Microbe Interactions of the BRIA UFRC RAS (Ufa, Russia). The colonies on potato dextrose agar (PDA) appear as abundant fluffy aerial mycelium in white and pinkish red tones. Red pigment is released into the medium over time. In present study, F. culmorum was grown on PDA ( $\mathrm{pH}$ 6.6) at $28^{\circ} \mathrm{C}$ for 5 days [14]. Inoculum of F. culmorum at a concentration of $10^{6}$ spores $/ \mathrm{mL}$ were prepared in distilled water with using a Goryaev chamber [14].

\subsection{Plant Materials and Growth Conditions}

The experiments were conducted on wheat plants (Triticum aestivum L., Cv. Omskaya 35). Before sowing, wheat seeds were sterilized in $96 \%$ ethanol for $1 \mathrm{~min}$, washed under running water until the smell of alcohol disappeared, and then were treated with B. subtilis $10-4\left(10^{5} \mathrm{CFU} \mathrm{mL}{ }^{-1}\right)$ [13], exogenous SA $(0.05 \mathrm{mM})$ [11], and B. subtilis $10-4\left(10^{5} \mathrm{CFU} \mathrm{mL}{ }^{-1}\right)+\mathrm{SA}(0.05 \mathrm{mM})$ by soaking the seeds into the solutions of B. subtilis 10-4, SA, B. subtilis 10-4 + SA, and water (control) for $1 \mathrm{~h}$. Thereafter, treated and non-treated seeds were grown on distilled water-moistened filter paper for 3 days under a $16 \mathrm{~h}$ light period $(12,000-16,000 \mathrm{lux})$ at a temperature of $20-22^{\circ} \mathrm{C}$. Next, the 3-day-old seedlings were isolated from the endosperm and transplanted into glasses with solutions of 12\% PEG-6000 (drought) or water (control) and left to grow for the next whole day. Thereafter, wheat seedlings were infected with the phytopathogenic fungus F. culmorum, which was carried out by introducing it into stems of 4-day-old seedlings of a suspension of conidia of the fungus in water $\left(10^{6}\right.$ spores $\left.\mathrm{mL}^{-1}\right)$. The incidence of 
disease development in wheat seedlings was assessed in 2, 4, 6, 10, and 14 days after F. culmorum infection. The changes in morpho-physiological and biochemical traits were assessed in 14-day-old seedlings (in 10 days after infection with F. culmorum and 11 days after exposure of drought (12\% PEG-6000)).

\subsection{Assessment of Plant Growth-Promoting (PGP) Traits of Strain 10-4 and Its Ability to Colonize Internal} Tissues of Wheat

Strain 10-4 was analyzed for PGP traits such as indole-3-acetic acid (IAA)-producing ability using LB medium containing L-tryptophan [15], siderophore production using plates with blue agar containing the dye Chrome Azurol S [16], and phosphate solubilization activity using Pikovskaya medium [17]. Nitrogen fixation of strain 10-4 was assayed using gas chromatography with a flame-ionization detector (Shimadzu GC-2014, Kyoto, Japan) [18]. Comparative analysis of B. subtilis 10-4, SA, and B. subtilis 10-4 + SA influence on the linear sizes of seedlings (root and shoot lengths) of wheat and fresh (FW) and dry biomass (DW) accumulation in them, both under normal growth conditions and the influence of F. culmorum, drought, and combined drought $+F$. culmorum stresses, were performed using classical physiological methods [19].

The ability of tested strain 10-4 to colonize the internal tissues of wheat plants was determined using surface-sterilized plants after inoculation with strain 10-4 in sterile conditions [13]. Segments of wheat leaves and roots were immersed in a $70 \%$ ethyl alcohol $(5 \mathrm{~min})$. Thereafter, alcohol was drained, and the segments were washed three times with sterile water and air-dried (10-15 min). Surface sterilized segments were laid out in Petri dishes with Bacillus ChromoSelect Agar (Sigma Aldrich, St. Louis, MO, USA) and kept $\left(24 \mathrm{~h}\right.$ at $\left.26^{\circ} \mathrm{C}\right)$ for bacterial growth. Using RAPD-PCR analysis, we determined the identity of bacteria grown around the segments of wheat leaves and roots with origin 10-4 (applied for inoculation of seeds before sowing) [13]. Isolation of bacterial DNA was carried out using lysis buffer ( $1 \%$ Tween-20, 1\% tryptone $\times 100,0.005 \%$ cresol red, $1 \%$ Chelex-100 (Bio-Rad, Hercules, CA, USA), and water) [13]. According to the results of RAPD-PCR of total DNA using AFK primers (50-GCGTCCATTC-30), we evaluated the strain genetic polymorphism. PCR amplification was carried out on Tercik equipment (DNA-Technology, Moscow, Russia). RAPD analyses were performed using horizontal electrophoresis in 1.5\% agarose gel in a SE-2 chamber (Helikon, Moscow, Russia) ( $25 \mathrm{kV}$ for $1 \mathrm{~h}$ ). Ethidium bromide was used to stain the gel, and the results were recorded using the Gel Doc XR system (Bio-Rad, Hercules, CA, USA).

\subsection{In Vitro Assay of Anti-Fungal Potency of B. subtilis 10-4 Against F. culmorum}

Anti-fungal potency of tested B. subtilis 10-4 was detected using the coculture of bacterial strain and F. culmorum previously plated on Petri dishes with PDA [14] (the antifungal potency of B. subtilis 10-4 in mix with SA was evaluated in PDA medium with addition of SA $(0.05 \mathrm{mM})$ ). To assess the antifungal potency, we performed an agar well diffusion assay. Bacterial culture medium aliquot $(150 \mu \mathrm{L})$ was filled in a well (5 mm diameter) made in PDA (pH 7.0), previously plated with a spore suspension of F. culmorum, and incubated at $30^{\circ} \mathrm{C}$ for 3 days. Thereafter, the zone of fungal growth inhibition was assessed. Inhibition ratio $(\%)=(C-E) / C \times 100 \%$, where $C$ is the diameter of the control colony and $\mathrm{E}$ is the diameter of the treatment colonies. To obtain metabolites of B. subtilis 10-4, we added the bacterial cell culture aliquot $(150 \mu \mathrm{L})\left(10^{8} \mathrm{CFU} \mathrm{mL}{ }^{-1}\right)$ into $15 \mathrm{~mL}$ of fresh LB medium, which was incubated at $30^{\circ} \mathrm{C}$ for 2 days (with $180 \mathrm{rpm}$ shaking) and centrifugated for $45 \mathrm{~min}$ at $5000 \times g$, and the cell-free supernatant was then used for assessment of the antifungal activity against F. culmorum in PDA plates, as mentioned above. Each experiment was performed in at least three replicates. The pathogen mycelium growth was visualized using a scanning electron microscope (SEM) Biozero BZ-8100E (Keyence Co., Osaka, Japan).

\subsection{Evaluation of F. culmorum-Caused Root Rot Development in Plants}

Disease development intensity was evaluated by visual symptoms on a five-point scale ( 0 -no symptoms, 1-damage from 1 to $25 \%$, 2-from 26 to 50\%, 3-from 51 to $75 \%$, 4-more than $75 \%$, 5-dead plant). In each variant, we used 50 seedlings in three replicates [20]. 


\subsection{Estimation of Lipid Peroxidation (Malondialdehyde (MDA)) in Wheat Seedlings}

MDA was estimated according to the Health and Packer method [21]. Fresh plant tissues (0.2 g) were homogenized with $1 \mathrm{~mL}$ of $10 \%$ trichloroacetic acid and centrifuged (10,000 rpm for $10 \mathrm{~min})$. Further, $1 \mathrm{~mL}$ of the supernatant was mixed with $20 \%$ trichloroacetic acid containing $0.25 \%$ thiobarbituric acid and was heated $\left(95^{\circ} \mathrm{C}\right.$ for $\left.30 \mathrm{~min}\right)$, quickly cooled using an ice bath, and centrifuged $(10,000 \mathrm{rpm}$ for $10 \mathrm{~min}$ ). The supernatant absorbance was measured at 532 and $600 \mathrm{~nm}$ using a SmartSpec Plus spectrophotometer (Bio-Rad, Hercules, CA, USA). The concentration of MDA was calculated using an extinction coefficient of $155 \mathrm{mM}^{-1} \mathrm{~cm}^{-1}$.

\subsection{Estimation of Proline (Pro) Concentration in Wheat Seedlings}

Pro was estimated according to the method of Bates et al. [22]. Fresh plant tissues $(0.5 \mathrm{~g})$ were extracted with $2.5 \mathrm{~mL}$ of boiled water. Further, $2.0 \mathrm{~mL}$ of obtained extract was mixed with an equal volume of ninhydrin solution $(1.25 \mathrm{~g}$ ninhydrin dissolved in $30 \mathrm{~mL}$ of glacial acetic acid and $20 \mathrm{~mL}$ of $6 \mathrm{M}$ phosphoric acid) and glacial acetic acid. Thereafter, the samples were incubated at $100{ }^{\circ} \mathrm{C}$ for $1 \mathrm{~h}$, cooled in an ice bath, and Pro was measured at $522 \mathrm{~nm}$ using a SmartSpec Plus spectrophotometer (Bio-Rad, Hercules, CA, USA).

\subsection{Statistical Analysis}

All of the microbiological, morpho-physiological, and biochemical experiments were carried out for at least three biological and three analytical replicates. The data were presented as the mean \pm standard error of the mean (SEM). Statistically significant differences between the mean values were evaluated using a two-way analysis of variance (ANOVA), followed by the Tukey test $(p<0.05)$.

\section{Results}

\subsection{Plant Growth-Promoting Traits of B. subtilis 10-4 and Its Ability to Colonize Internal Tissues of Wheat}

It was revealed that pre-sowing inoculation with tested B. subtilis 10-4 visibly promoted growth of wheat plant under normal conditions (Figure 1). Seed germinations (3 days after bacterial inoculation and seed sowing) increased up to $20 \%$ (from $79 \%$ in non-inoculated control to $98 \%$ in inoculated) (Figure 1A). Lengths of roots and shoots of 6-day-old seedlings increased up to $20-30 \%$ in comparison to the control (Figure 1B). An increase in fresh and dry biomass accumulation (up to 15-18\%) in seedlings under the influence of B. subtilis 10-4 was also observed (Figure 1C).

Furthermore, the ability of tested strain 10-4 to produce compounds most likely contributing to enhancing plant growth processes such as siderophores and indole-3-acetic acid (IAA), and fixing atmospheric nitrogen (Table 1) was revealed. In particular, using the purified bacterial strain 10-4 freshly grown in LB medium after transferring in plates with blue agar containing the dye Chrome Azurol $S$ and LB medium containing L-tryptophan, we found the ability of tested strain 10-4 to produce siderophores and indole-3-acetic acid (IAA), respectively. The analysis was carried out using gas chromatography with a flame ionization detector, allowing for the detection of the ability of strain 10-4 to fix atmospheric nitrogen. With that, there was no detected ability of strain 10-4 to phosphate solubilization in Pikovskaya medium (Table 1). 
A

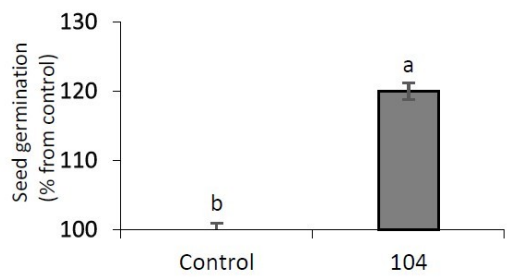

C

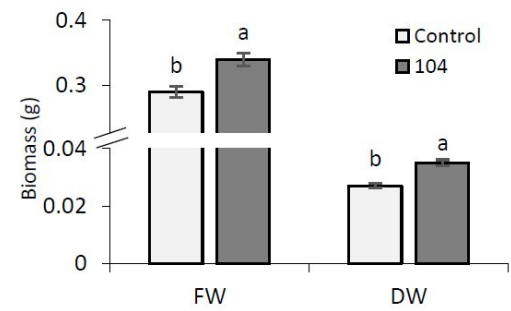

B

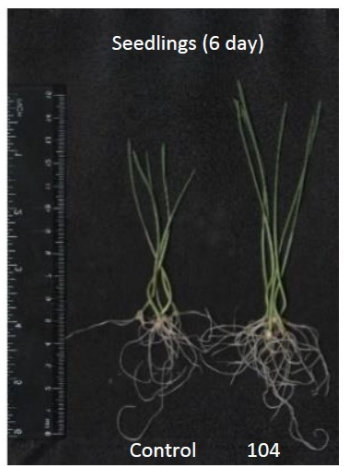

Figure 1. Effect of Bacillus subtilis 10-4 (104) on the percentage of seed germination (3 days after sowing) (A), length of seedlings (roots and shoots) (B), and fresh (FW) and dry biomass (DW) accumulation (C) (6 days after sowing) under normal growth conditions. The error bars show the average of three replicates \pm standard error of the mean (SEM) $(n=50)$. Different letters indicate a significant difference between the average values for $p<0.05$.

Table 1. Some plant growth-promoting (PGP) traits of tested bacteria B. subtilis 10-4.

\begin{tabular}{ccccc}
\hline Bacteria & IAA $\left(\mathrm{mg} \mathrm{L}^{-1}\right)$ & Siderophore Production $(\mathbf{c m})$ & p Solubilization $\left(\mathrm{mg} \mathrm{L}^{-1}\right)$ & Nitrogen Fixation $(\mu g$ N2/mL/h) \\
\hline B. subtilis 10-4 & $5.8 \pm 0.2$ & $1.2 \pm 0.1$ & - & $0.08 \pm 0.02$ \\
\hline
\end{tabular}

Using surface-sterilized 3-day-old wheat seedlings non-inoculated (control) and pre-inoculated before sowing with $B$. sibtilis 10-4 (test) and RAPD-PCR analysis, we showed the ability of B. subtilis 10-4 to colonize internal tissues of wheat (Figure 2). Around plant segments inoculated with B. subtilis 10-4 and incubated in Bacillus ChromoSelect Agar, we observed the growth endophytic bacteria belonging to genera B. subtilis (light green to green colonies), while bacterial growth was absent around non-inoculated plant segments (Figure 2A). The comparison of the original applied bacteria B. subtilis 10-4 used for plant inoculation and bacteria grown around wheat plant segments using RAPD-PCR analysis allowed for the confirmation of their identity (i.e., B. subtilis 10-4 as endophytic bacteria) (Figure 2B).

A
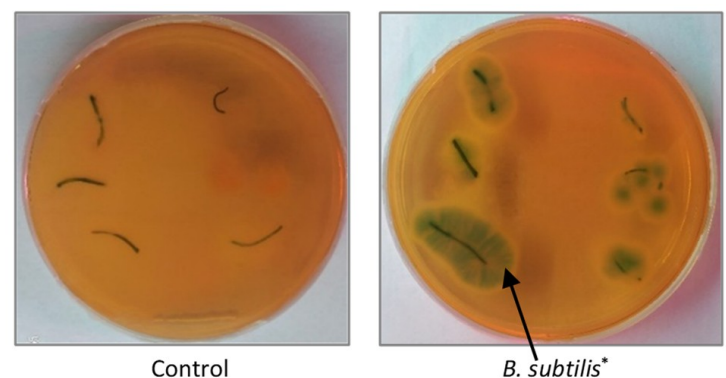

B

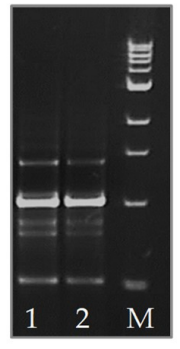

Figure 2. The ability of B. subtilis 10-4 to colonize the internal tissues of 3-day-old wheat seedlings after pre-sowing inoculation of seeds. (A) The absence of bacterial growth around the surface-sterilized root and leaf segments of non-inoculated wheat seedlings (control) and bacterial growth $\left({ }^{*}\right.$ light green to green colonies) around the surface-sterilized leaf and root segments of wheat seedlings pre-inoculated with B. subtilis 10-4 (B. subtilis). (B) PAAG electropherogram after RAPD-PCR analysis: 1-DNA of origin B. subtilis 10-4 used for pre-sowing inoculation of seeds; 2-DNA of bacteria isolated from the bacteria growing around wheat segments (inoculated with 10-4 before sowing of seeds) in Bacillus ChromoSelect Agar (Sigma Aldrich, St. Louis, MO, USA); M — DNA marker (New England BioLabs, UK). 


\subsection{In Vitro Antagonistic Potency of B. subtilis 10-4 against F. culmorum}

In vitro studies demonstrated that endophytic bacteria B. subtilis $10-4$ have antagonistic activity against F. culmorum (Figure 3A). The studies showed that F. culmorum mycelium diameter was decreased with increase in concentration of B. subtilis 10-4 in plates with PDA, leading to a gradual increase in inhibition ratio of F. culmorum (Table 2). The inhibition ratio of F. culmorum reached $67.9 \%$ at $10^{8}$ CFU mL $\mathrm{mL}^{-1}$ of $B$. subtilis 10-4. Moreover, it was revealed that the addition of SA to the PDA medium increased the ability of B. subtilis 10-4 to suppress the development of $F$. culmorum, reaching $73.7 \%$ at $10^{8} \mathrm{CFU} \mathrm{mL} \mathrm{mL}^{-1}$ of B. subtilis 10-4 (Table 2). The microscopic observation of the F. culmorum mycelia showed morphological variations. F. culmorum mycelia were well organized in the area of the B. subtilis 10-4 bacterial culture medium absence (Figure 3B), while numerous fungal mycelia gaps appeared in the area of presence of B. subtilis 10-4 bacterial culture medium (Figure 3B). As shown in further analysis, the metabolite syntheses by B. subtilis 10-4 effectively suppressed F. culmorum growth in vitro in PDA plates (Figure 3C), indicating their contribution in the detected antifungal activity of B. subtilis 10-4 bacterial culture against $F$. culmorum.

A

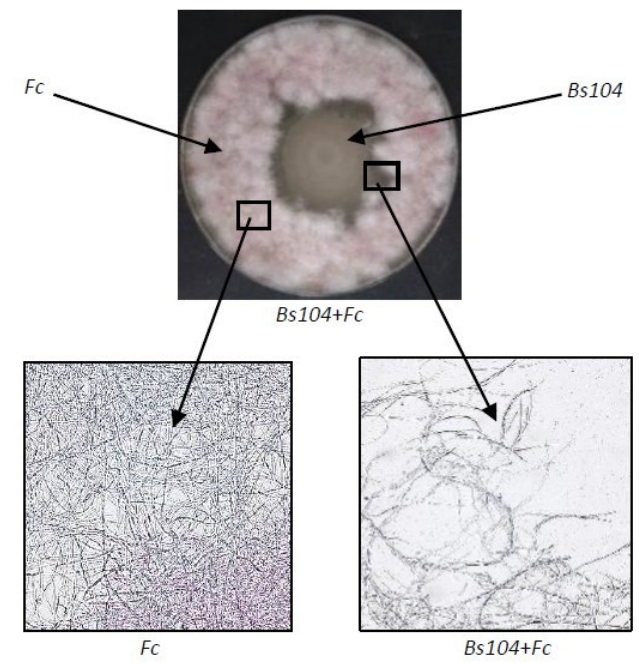

C
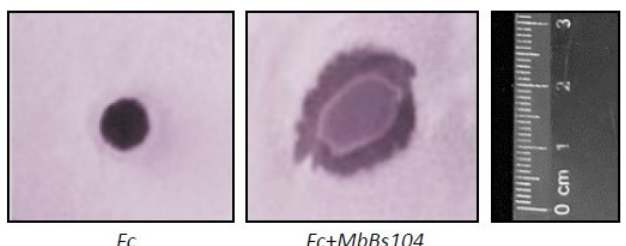

Figure 3. In vitro antagonistic activity of B. subtilis 10-4 (Bs104) against Fusarium culmorum on potato dextrose agar (PDA) plates at 3 days after incubation at $30^{\circ} \mathrm{C}(F c)(\mathbf{A})$; microscopic visualizations of the growth of $F c$ and its morphology in the absence (B) and on the border with B. subtilis 10-4 (C). In vitro antagonistic activity of metabolites synthesized by B. subtilis 10-4 (MbBs104) against F. culmorum ( Fc) (D). The observation was made using a scanning electron microscope (Biozero BZ-8100E (Keyence Co., Osaka, Japan)). Fc—F. culmorum; Bs104—B. subtilis 10-4; MbBs104—metabolites synthesized by B. subtilis $10-4$.

Table 2. The inhibitory effect of B. subtilis 10-4 (Bs104) and B. subtilis 10-4 + salicylic acid (SA) on the growth of F. culmorum mycelium.

\begin{tabular}{|c|c|c|c|c|c|c|c|}
\hline Bs104 (CFU mL $\left.{ }^{-1}\right)$ & 0 & $10^{3}$ & $10^{4}$ & $10^{5}$ & $10^{6}$ & $10^{7}$ & $10^{8}$ \\
\hline Inhibition ratio * $(\%)$ & 0 & 58.3 & 60.8 & 62.7 & 64.1 & 65.4 & 67.9 \\
\hline Bs104 (CFU mL $\left.{ }^{-1}\right)+$ SA $(0.05 \mathrm{mM})$ & 0 & $10^{3}$ & $10^{4}$ & $10^{5}$ & $10^{6}$ & $10^{7}$ & $10^{8}$ \\
\hline Inhibition ratio * $(\%)$ & 0 & 62.1 & 65.5 & 68.6 & 70.7 & 72.2 & 74.7 \\
\hline
\end{tabular}

${ }^{*}$ Colony radius was measured after 3 days of incubation at $30^{\circ} \mathrm{C}$. 
3.3. Effect of B. subtilis 10-4, SA, and B. subtilis 10-4 + SA on F. culmorum-Instigated Root Rot Development in Wheat Seedlings Under Normal and Combined Drought Conditions

Artificial infection of plants with the phytopathogenic fungus F. culmorum under normal growth conditions led to a gradual strengthening of the symptoms of Fusarium root rot (FRR) damage, reaching $60 \%$ or more (Figure $4 \mathrm{~A}$ ). Light brown and brown traces of lesions became noticeable on the roots and root zone of the shoots. However, it should be noted that the initial stages of observation, the disease did not appear, and the symptoms became noticeable only 10-14 days after infection. Application of B. subtilis 10-4 (10 $\left.\mathrm{CFU} \mathrm{mL}^{-1}\right)$, SA $(0.05 \mathrm{mM})$, and B. subtilis $10-4+$ SA facilitated a significant reduction (by 6.5-7.5 times) in the development of FRR, up to a complete absence of symptoms (for B. subtilis 10-4 +SA). Thus, upon inoculation with B. subtilis 10-4, the degree of FRR development was significantly lower (in comparison with control) and did not exceed 5-10\% (Figure 4A). These data correlate with the data obtained above regarding the detected in vitro antagonistic activity of tested bacteria B. subtilis 10-4 against F. culmorum (Figure 2). At the same time, when B. subtilis 10-4 was applied together with SA, the symptoms of FRR in wheat seedlings were completely absent, which indicates the contribution of exogenous SA to enhancing the antagonistic potential of strain 10-4 against F. culmorum - the causative agent of FRR (Figure 4A).
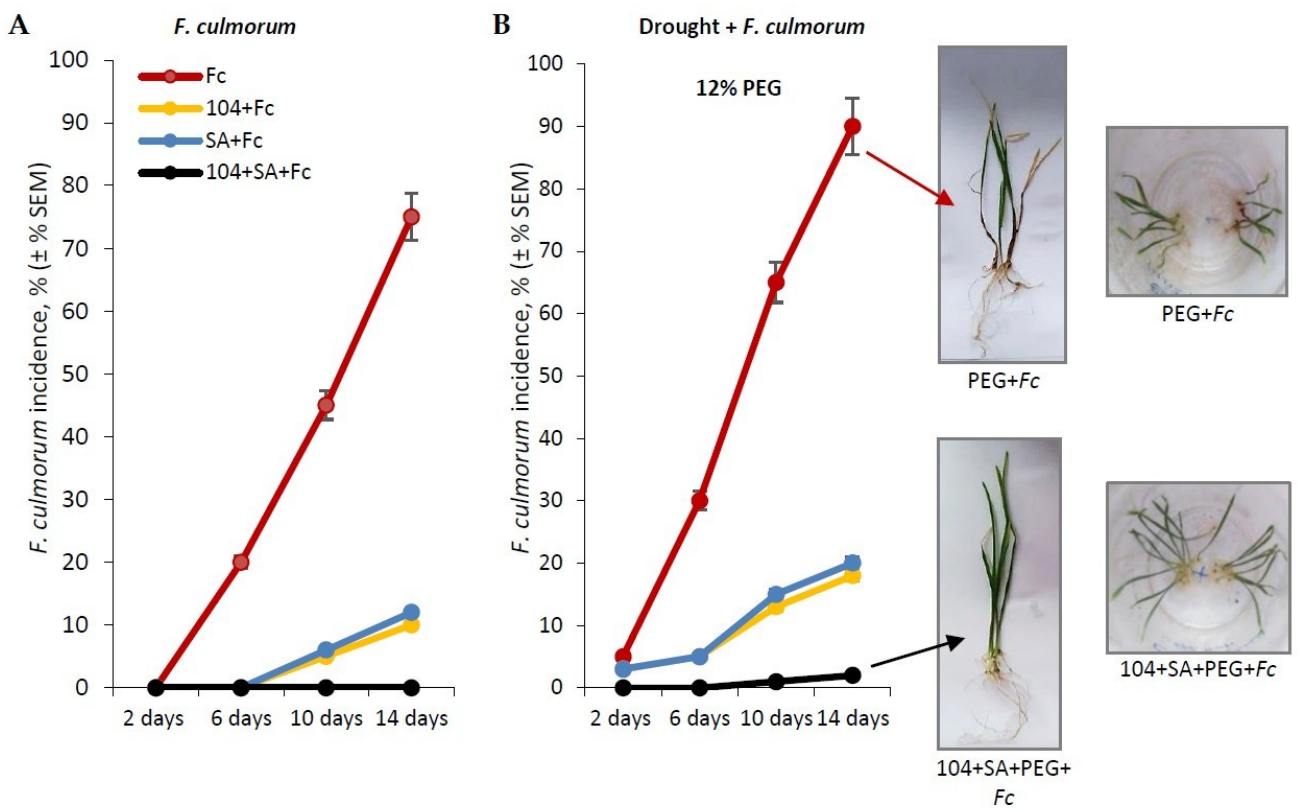

Figure 4. The influence of endophytic bacteria B. subtilis 10-4 (104) $\left(10^{5}\right.$ colony-forming units $\left.(\mathrm{CFU}) \mathrm{mL}^{-1}\right)$, salicylic acid (SA) $(0.05 \mathrm{mM})$, and composition of B. subtilis 10-4 + SA (104 + SA) on F. culmorum-instigated root rot $(F c)$ development in wheat plants under normal (A) and combined drought stress (12\% PEG-6000) $(\mathrm{PEG}+F c)$ conditions $(\mathbf{B})$. The error bars show the average of three repetitions $\pm \mathrm{SEM}(n=50)$.

It has been found that under drought conditions in non-inoculated control plants, infestation with F. culmorum along with traces of the defeat of the root part (which reached up $80 \%$ or more) resulted in wheat leaf yellowing (total number of yellowing plants was $10-15 \%(n=50)$ ) (Figure 4B). This, obviously, can be associated with the additional stressful effects of drought and a sharp violation of water metabolism. From this, the leaves began to turn yellow, starting from the top. As for the plants pre-treated with $B$. subtilis 10-4, SA, and B. subtilis 10-4 + SA, the visual symptoms of F. culmorum development was notably lower than in the control. B. subtilis 10-4 and SA reduced FRR development by 4.5-5 times (Figure 4B). With that, when B. subtilis 10-4 or SA were used separately, a slight yellowing of the upper parts of the leaves appeared. Thus, the total damaged leaves were about $2 \%$ for SA and $0.2-0.5 \%$ for $B$. subtilis $10-4$. Therefore, under the combined effects of drought and the causative agent 
of FRR, the maximum positive effect on wheat plants had a composition B. subtilis 10-4 + SA. In plants pre-treated with B. subtilis 10-4 + SA, there were almost no symptoms of the disease and the effect of drought (Figure 4B). Moreover, the seedlings were characterized by increased turgor and were much stronger in contract with both non-inoculated control plants and plants that were treated with SA or B. subtilis $10-4$ separately.

\subsection{Effect of B. subtilis 10-4, SA, and B. subtilis 10-4 + SA on Growth of Non-Infected and F.} culmorum-Infected Wheat Plants under Normal and Combined Drought Conditions

It was revealed that under normal growth conditions, pre-sowing treatment of seeds with $B$. subtilis 10-4, SA, and composition of B. subtilis 10-4 + SA increased (in different levels) healthy (non-infected) wheat plant growth (shoot and root lengths, fresh weight (FW) and dry weight (DW) biomasses), with the most pronounced positive effect being after application of composition B. subtilis 10-4 + SA (Figure 5A,B).

A

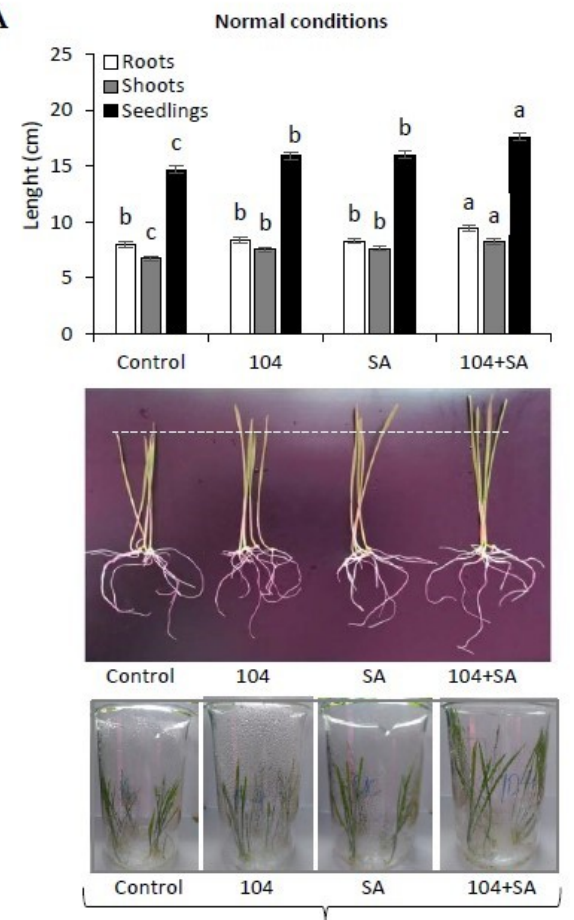

B

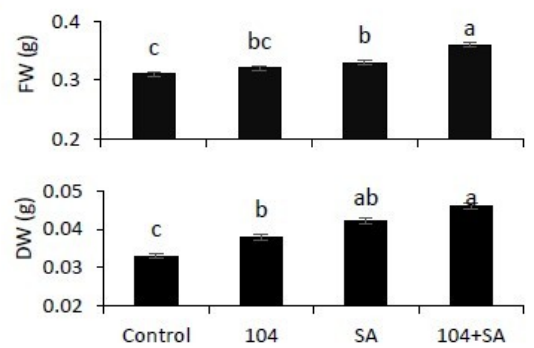

C
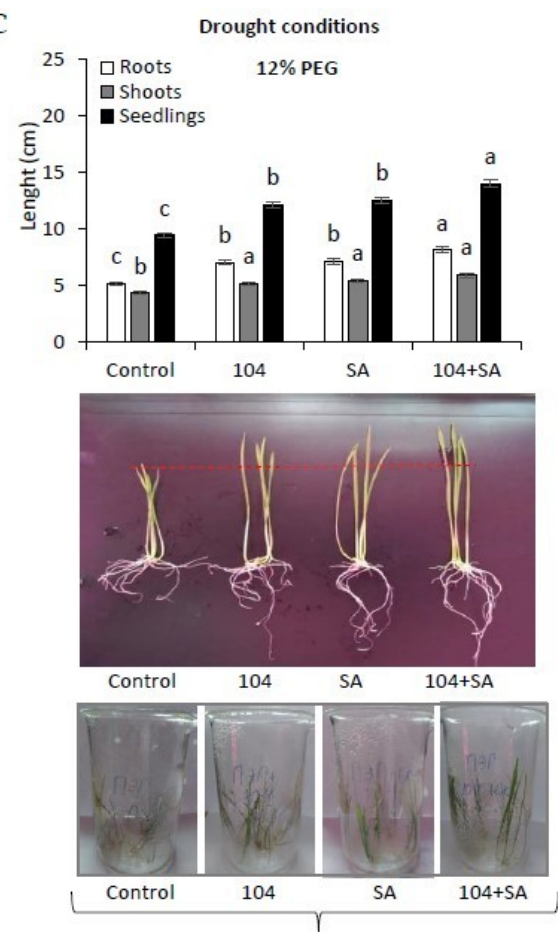

D

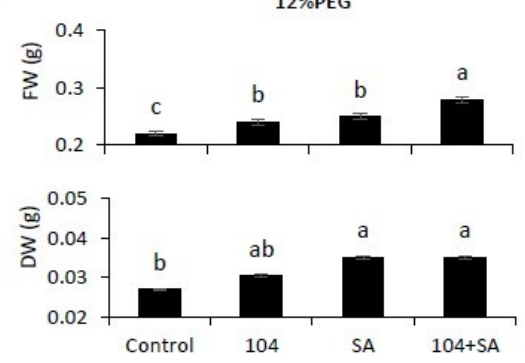

Figure 5. Effect of endophytic B. subtilis 10-4 (104) $\left(10^{5} \mathrm{CFU} \mathrm{mL}{ }^{-1}\right)$, SA $(0.05 \mathrm{mM})$, and composition of B. subtilis 10-4 + SA (104+SA) on the linear dimensions (root and shoot lengths) and fresh weight (FW) and dry weight (DW) biomass accumulation of wheat seedlings under normal (A,B) and drought stress (12\% PEG) conditions (C,D). Fourteen-day-old seedlings; drought stress (12\% PEG) exposure time-11 days. Control—plants without treatments; 104, SA, and 104+SA-plants pre-treated with B. subtilis 10-4, SA, and B. subtilis 10-4 + SA, respectively. The error bars show the average of three replicates $\pm \operatorname{SEM}(n=50)$. Different letters indicate a significant difference between the average values for $p<0.05$. 
Application of B. subtilis 10-4, SA, and B. subtilis 10-4 + SA contributed to the activation of wheat seedling growth, which was manifested in increasing the linear dimensions (root and shoot lengths) of plants up to $25-50 \%$, depending on the treatment option, with a maximum growth-promoting effect when the strain 10-4 was combined with SA (increase the length of seedlings up to 40-50\%) (Figure 5A). Exposure to drought (12\% PEG-6000) led to significant inhibition of growth processes and a decrease (by $50 \%$ or more) in the linear dimensions of plant roots and shoots (Figure $5 \mathrm{C}$ ). In particular, the length of the seedlings reduced from 14.7 (control) to $9.4 \mathrm{~cm}$ (drought). With that, treatment with B. subtilis 10-4, SA, and B. subtilis 10-4 + SA softened (to varying degrees, about $15-40 \%$ ) the degree of damage effect of drought on plant growth, which was reflected in higher indices of the root and shoot lengths of plants as compared with the non-treated control. Moreover, the greatest protective effect from the damaging effect of drought on wheat plant growth was observed with the joint use of strain B. subtilis 10-4 with SA (Figure 5C). A similar characteristic of the influence of B. subtilis 10-4, SA, and B. subtilis 10-4 + SA was revealed when assessing the accumulation FW and DW of seedlings both under normal and drought stress conditions (Figure 5B,D). These data suggest that the most effective way of stimulating growth processes and protecting wheat plants against the negative impact of drought is the application of B. subtilis 10-4 in composition with SA, since this combination had a maximum positive effect on growth indicators (including growth in the lower (roots) and above-ground (shoots) tissues, as well as the accumulation of fresh and dry biomasses) of wheat, under both normal and drought conditions.

The infestation of plants with F. culmorum, both under normal and especially under combined drought stress conditions, led to a significant reduction of wheat plant growth processes and biomass accumulation (Figure 6), in contrast with non-infected control ones (Figure 5). Application of B. subtilis 10-4, SA, and B. subtilis 10-4 + SA alleviated the F. culmorum-instigated negative effect of plant growth (length of roots and shoots) both under normal (Figure 6A) and combined drought conditions (Figure 6B). Thus, under the influence of B. subtilis 10-4, SA, and especially under B. subtilis 10-4 + SA, the length of wheat seedlings was higher (up to 15-30\%) than in non-treated control plants infected with F. culmorum and growing under non-drought (Figure 6A) conditions. While under combined F. culmorum and drought stresses, the application of B. subtilis 10-4, SA, and B. subtilis 10-4 + SA also showed a protective effect on wheat seedling growth, but it was less pronounced (Figure 6B) than under only one individual stress influence (Figure 6A). With that, under combined F. culmorum and drought stresses, the most positive effect on wheat growth processes was observed when $B$. subtilis 10-4 was used in composition with SA. Thus, in plants treated with B. subtilis 10-4 + SA, the length of seedlings (roots, shoots) was higher by $10-20 \%$ than in the control (Figure $6 \mathrm{~B}$ ). Plants pre-treated with B. subtilis 10-4, SA, and B. subtilis 10-4 + SA also showed better DW and FW biomass accumulation both under F. culmorum infestation (Figure 6C) and combined drought and F. culmorum stresses (Figure 6C), with the most pronounced positive effects after application of B. subtilis 10-4 together with SA.

Thus, the obtained data on the effect of the studied endophytic bacteria B. subtilis $10-4$ alone and in composition with SA on the development of symptoms of FRR caused by phytopathogenic fungus F. culmorum and the intensity of plant growth processes under normal (non-drought) and drought stress conditions is in favor of the most effective use of the B. subtilis 10-4 + SA composition, both in stimulating growth and protecting plants against the damaging impact of combined abiotic/biotic stresses. The findings allow for the supposition that such physiological actions of endophytic B. subtilis 10-4 alone and in composition with SA may be connected with several possible mechanisms, which require further thorough investigations to fully exploit the potential of these biological agents as a part of organic agriculture to improve wheat resistance/tolerance and productivity. 
A
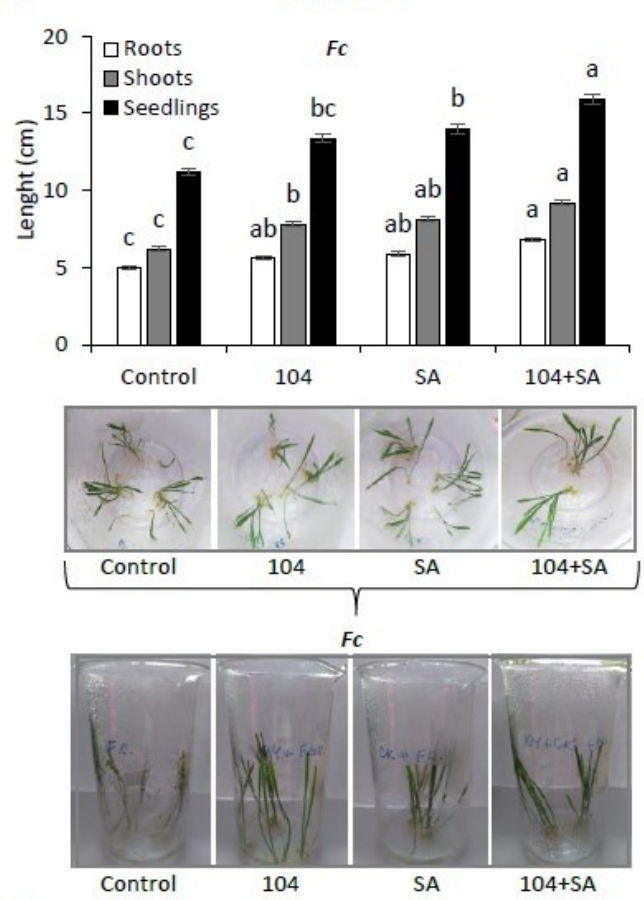

C
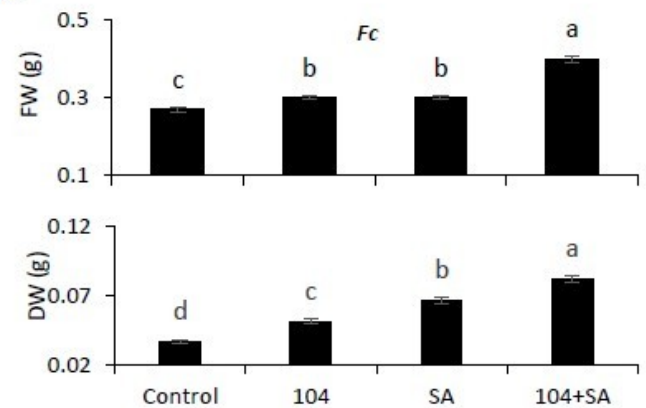

B
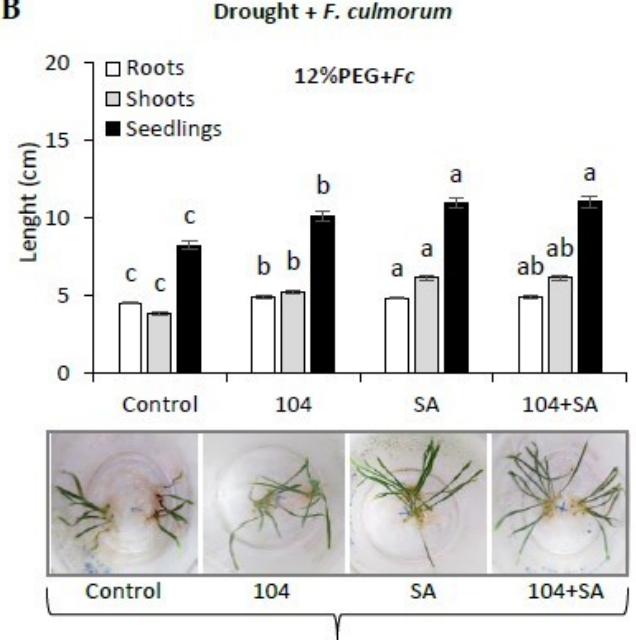

$12 \%$ PEG + FC

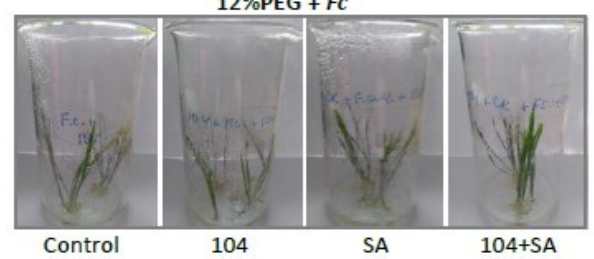

D
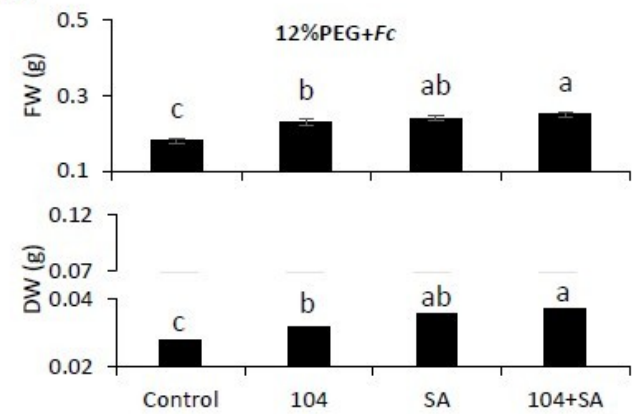

Figure 6. Effect of B. subtilis 10-4 (10 $\left.\mathrm{CFU} \mathrm{mL}^{-1}\right)$, SA $(0.05 \mathrm{mM})$, and composition of B. subtilis 10-4 $+\mathrm{SA}$ on growth of wheat seedlings and their fresh weight (FW) and dry weight (DW) accumulation under the influence of F. culmorum $(F c)(\mathbf{A}, \mathbf{C})$ and combined drought $+F$. culmorum (Drought $+F c$ ) (B,D) stresses. Fourteen-day-old seedlings; drought stress (12\% PEG) exposure time-11 days; time after infection with F. culmorum-10 days. Control-plants without treatments; 104, SA, and 104+SA-plants pretreated with B. subtilis 10-4, SA, and B. subtilis 10-4 + SA, respectively. The error bars show the average of three repetitions \pm SEM $(n=50)$. Different letters indicate a significant difference between the average values for $p<0.05$.

3.5. Effect of B. subtilis 10-4, SA, and B. subtilis + SA on Lipid Peroxidation (MDA) of F. culmorum-Infected Wheat Plants under Normal and Combined Drought Conditions

It was found that artificial infection with the phytopathogenic fungus F. culmorum led to an increase of MDA concentration in wheat plants, both during cultivation under normal (about two times) and especially under combined drought stress (about 3.5-4 times) conditions (Figure 7). Thus, under normal growth conditions, infestation with F. culmorum increased MDA in control plants by about two times, whereas under combined drought stress in F. culmorum-infected plants, the content of MDA increased more notably. At the same time, pre-sowing treatment of seeds with B. subtilis 10-4, alone and in a mix with SA, facilitated the reduction of the pathogen-instigated increase of MDA content in seedlings, both under normal and combined drought stress conditions. In particular, B. subtilis 10-4, SA, and B. subtilis 10-4 + SA reduced drought-initiated MDA accumulation in healthy (non-infected) 
plants by approximately 1.5, 1.8, and 2.4 times, respectively (Figure 7). It should be noted that in healthy (non-infected) plants grown under normal conditions that were pre-treated with B. subtilis 10-4, we also observed a slight increase in MDA content, while for SA and B. subtilis 10-4 + SA, there were no statistically significant differences compared to the control.

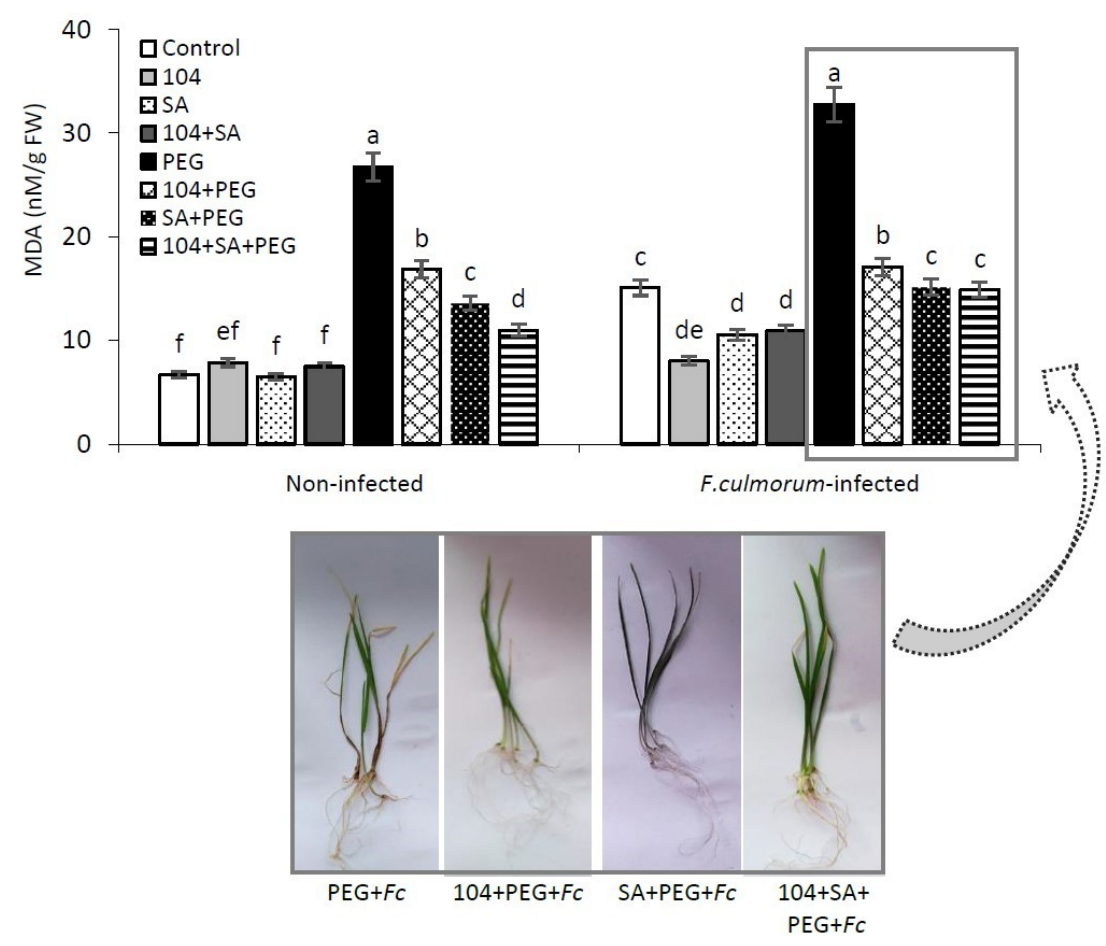

Figure 7. Effect of B. subtilis 10-4 (104) $\left(10^{5} \mathrm{CFU} \mathrm{mL}^{-1}\right)$, salicylic acid (SA) (0.05 mM), and composition of B. subtilis 10-4 + SA (104+SA) on the concentration of malondialdehyde (MDA) in non-infected by F. culmorum (non-infected) and infected by F. culmorum (F. culmorum-infected) wheat plants under normal and combined drought stress (PEG) conditions. Fourteen-day-old seedlings; drought stress (12\% PEG) exposure time-11 days; time after infection with F. culmorum-10 days. Fc-F. culmorum. The error bars show the average of three replicates \pm SEM. Different letters indicate a significant difference between the average values for $p<0.05$.

Infection of plants with F. culmorum resulted in twofold MDA accumulation (compared with the control), whereas B. subtilis 10-4, SA, and B. subtilis 10-4 + SA contributed to decreasing such F. culmorum-induced MDA accumulation by 1.8, 1.8, and 1.5 times, respectively (Figure 7). Influence of combined drought and F. culmorum infection increased MDA in plants by approximately 4 times (in comparison with healthy control plants growing under normal conditions) and 2.1 times (in comparison with F. culmorum-infected plants growing under non-drought conditions) (Figure 7). At once, plants pre-treated with B. subtilis 10-4, both alone and in composition with SA, significantly decreased (by 1.9-2.1 times) combined drought and F. culmorum-instigated lipid peroxidation (MDA) in plants under the same growth conditions.

3.6. Influence of B. subtilis 10-4, SA, and B. subtilis 10-4 +SA on Proline (Pro) Accumulation in F. culmorum-Infected and Non-Infected Wheat Seedlings under Normal and Combined Drought Conditions

Our results showed that the application of B. subtilis 10-4, SA, and B. subtilis 10-4 + SA resulted in a decrease (by 1.6-1.9 times) of drought-induced Pro accumulation in wheat plants (Figure 8), whereas under normal conditions we observed no significant difference from the control upon B. subtilis 10-4, SA, and B. subtilis 10-4 + SA treatments. In plants infected with F. culmorum and growing under normal (non-drought) growth conditions, there was practically no significant difference from the control for SA and B. subtilis 10-4 + SA, except for B. subtilis 10-4, where the content of Pro increased by about 
1.2 times. It was found that the combined influence of drought and F. culmorum infection led to a significant increase (by up to three or more times) in the content of Pro in wheat plants (Figure 8). At the same time, pre-treatment with B. subtilis 10-4, SA, and the composition of B. subtilis 10-4+ SA contributed to a decrease of such Pro accumulation. Thus, B. subtilis 10-4, SA, and B. subtilis 10-4 + SA decreased combined drought and F. culmorum-instigated Pro content by 1.8, 2.2, and 1.6 times, respectively.

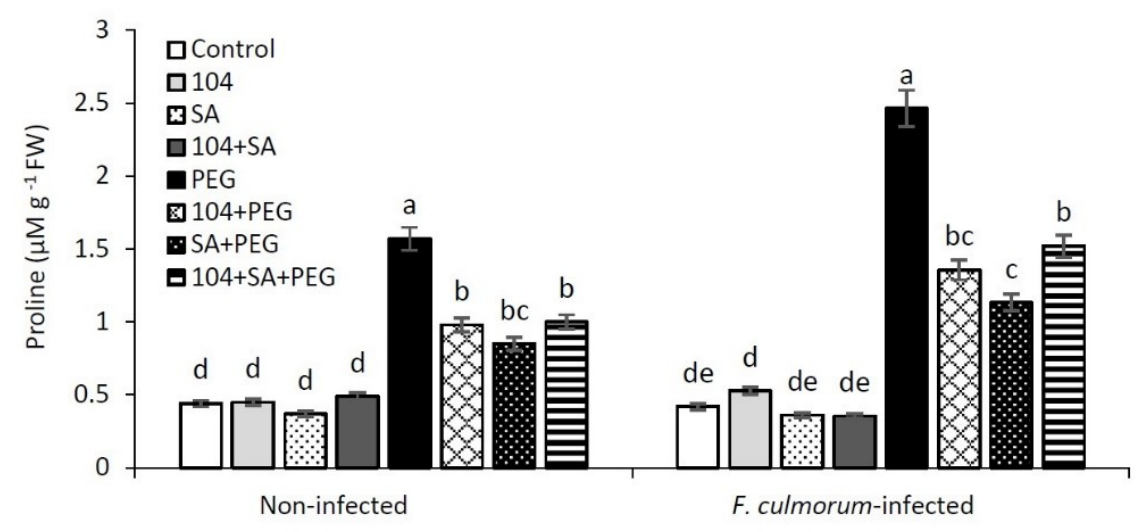

Figure 8. Effect of B. subtilis 10-4 (104) (105 CFU mL $\left.{ }^{-1}\right)$, salicylic acid (SA) (0.05 mM), and B. subtilis 10-4 + SA (104+SA) on the content of osmoprotectant proline in non-infected wheat plants and wheat plants infected with F. culmorum (F. culmorum-infected) under normal and drought conditions. Fourteen-day-old seedlings; drought stress (12\% PEG) exposure time-11 days; time after infection with F. culmorum-10 days. The error bars show the average of three replicates \pm SEM. Different letters indicate a significant difference between the average values for $p<0.05$.

Thus, the obtained data on the effect of the studied endophytic bacteria B. subtilis 10-4 (capable of colonizing the internal tissues of wheat plant as well as producing IAA, siderophores, and fixed nitrogen), separately and in composition with SA, on the development of F. culmorum-instigated root rot symptoms and the intensity of plant growth processes under normal and combined drought stress was indicated as being in favor of the most effective use of the B. subtilis 10-4 + SA composition, both to improve wheat growth stimulation and tolerance of wheat plants under combined drought and FRR stresses.

\section{Discussion}

Our results demonstrated that tested $B$. subtilis 10-4 effectively colonize the internal tissues of wheat plants (Figure 2) and are capable of producing IAA, siderophores, and fixed nitrogen (Table 1), as well as enhancing wheat plant growth both under normal (Figures 1 and 5A), drought (Figure 5B), FRR, and combined drought + FRR stresses (Figure 6). The results showed that artificial infection of wheat plants with F. culmorum under normal growth conditions led to a gradual increase in FRR damage symptoms, reaching $60 \%$ or more (Figure $4 \mathrm{~A}$ ). This, in general, is consistent with the literature data that root rot is outwardly subtle but is the most common and harmful disease of cereals, including wheat. Crop losses from FRR can be significant, reaching in some years up to 25\% or more [3]. When plants are affected by FRR, growth inhibition, disturbance in the dynamics of plant organogenesis, the impaired formation of all systemically important elements of the crop structure, and a significant decrease in product quality and its possible contamination with phytopathogenic toxins occur $[3,4]$. Here, we revealed B. subtilis 10-4, SA, and B. subtilis 10-4 + SA facilitated to a significant reduction (by $50-60 \%$ ) of FRR incidence, up to a complete absence of symptoms (for B. subtilis 10-4 + SA) under normal (non-drought) growth conditions (Figure 4A). This data correlates with the data obtained above regarding detected in vitro anti-fungal activity of B. subtilis 10-4 against F. culmorum (Figure 2). At the same time, when $B$. subtilis 10-4 was applied together with SA, the symptoms of FRR in wheat 
seedlings were completely absent, which indicates the contribution of exogenous SA to enhancing the antagonistic potential of strain 10-4 against FRR (Figure 4A). These data demonstrated the synergetic positive effect of SA. Previously, we obtained the data about the ability of SA to increase potato resistance against postharvest disease caused by F. oxysporum and Phytophthora infestans [20,23].

Under drought conditions in non-inoculated control plants, infestation with F. culmorum, along with traces of the defeat of the root part (which reached up $80 \%$ or more), resulted in wheat leaf yellowing (total number of yellowing plants was 10-15\% $(n=50)$ ) (Figure 4B). From this, the leaves began to turn yellow, starting from the top. This, obviously, can be associated with the additional stressful effects of drought and a sharp violation of water metabolism [2,7]. The obtained data indicate that the interaction between these two stresses negatively affects plant growth. According to the literature data, co-existing drought can modulate the interaction of various pathogens and plants in different ways, which leads to either suppression or an increased growth of pathogens [7]. The findings on the influence of combination of abiotic and biotic stresses on plants indicate their synergistic effects [24]. Abiotic stresses can influence the interaction of plant and pathogen both negatively and positively, hereby decreasing or increasing the incidence of disease development. Thus, it is very important to investigate in detail the features of the interactions between abiotic and biotic stresses in order to better understand the mechanisms underlying on their impact on plants [24]. According to the modern concept based on the available data on the simultaneous influence of abiotic (including drought) and biotic stresses, the dominant role of abiotic stresses is indicated, which contributes to the intensification of the development of plant diseases [25-29]. For example, it has been shown that drought enhanced Rhizoctonia bataticola-initiated disease incidence in chickpea [7]. In other studies, the incidence of collar rot disease caused by Sclerotium rolfsii [30] and Rhizoctonia solani-instigated wet root rot [31] increased under high soil moisture conditions. Moreover, the interactions between plant, pathogen, and abiotic stresses may be more complicated under field conditions and may vary depending on several factors such as combinations of the soil microbiome and plant phytobiome, as well as climatic characteristics of the area of plant cultivation [7]. In addition, these multiple stressors can occur in different ways; in particular, two stresses can occur either simultaneously or sequentially [7]. With that, there is also reports of a decrease in the incidence of diseases caused by Botrytis cinerea and Oidium neolycopersici after drought stress exposure [32]. The possible result of combined stress exposure may also be that plants, defending themselves from one form of stress, may become more resistant to other types of stresses [33]. The manifestation by plants of phenomena such as cross-tolerance indicates that plants possessing a powerful regulatory system can quickly adapt to changing environmental conditions [24].

Our results showed that in plants pre-treated with B. subtilis 10-4, SA, and B. subtilis 10-4 + SA, the visual symptoms of F. culmorum development were notably lower than in the control. B. subtilis 10-4 and SA reduced FRR development by $4.5-5$ times (Figure 4 B). With that, when B. subtilis 10-4 or SA were used separately, a slight yellowing of the upper parts of the leaves appeared. Thus, the total damaged leaves was about $2 \%$ for SA and $0.2-0.5 \%$ for B. subtilis $10-4$. Therefore, under the combined effects of drought and the causative agent of FRR, the maximum positive effect on wheat plants had a composition B. subtilis 10-4 + SA. In plants pre-treated with B. subtilis 10-4 + SA, there were almost no symptoms of the disease and the effect of drought (Figure 4B). Moreover, the seedlings were characterized by increased turgor and were much stronger in comparison with both non-inoculated control plants and plants that were treated with SA or B. subtilis 10-4 separately. These data indicate that B. subtilis 10-4, both alone and in composition with SA, form wheat plant tolerance to combined drought and FRR. As a result of the complicated interaction between drought, wheat plant, pathogen, and endophytic B. subtilis 10-4 (B. subtilis 10-4 + SA), plants become resistant to FRR.

Since growth is an integral indicator of the degree of damaging effect of stress factors on plants, a comparative analysis of the intensity of growth processes (length of roots and shoots) and fresh and dry biomass accumulation was performed. It was found that the application of B. subtilis 10-4 alone and especially in composition with SA notably enhanced elongation of wheat seedlings (roots, shoots; up to $20-50 \%$ ) and their fresh and dry biomass accumulations under normal growth conditions 
(Figures 1 and 5A), exerting protective effects on these parameters under drought (Figure 5B), FRR, and combined drought + FRR stresses (Figure 6). In particular, exposure to drought (12\% PEG-6000) led to significant inhibition of growth processes and a decrease (by $50 \%$ or more) in the linear dimensions of plant roots and shoots (Figure 5C,D), while the damaging effect of F. culmorum both under normal (non-drought) and especially combined drought conditions were much more pronounced (Figure 6).

Simultaneously, treatment with B. subtilis 10-4, SA, and B. subtilis 10-4 + SA softened to varying degrees the level of the damaging effect of drought (up to 40\%) and combined drought + FRR (about $20 \%$ ) on plant growth, which was reflected in higher indices of plant root and shoot lengths compared to the non-treated control (Figures 5 and 6). Moreover, the greatest protective effect against the damaging effects of drought and combined drought + FRR stresses on plant growth was observed after the joint application of composition B. subtilis 10-4 + SA. A similar character of the influence of B. subtilis 10-4, SA, and B. subtilis 10-4 + SA was revealed when assessing the accumulation fresh and dry biomass of seedlings both under normal and combined drought + FRR stresses (Figures 5 and 6). These data suggest that the most effective means of stimulating growth processes and protecting wheat plants from the negative effects of drought, FRR, and combined drought + FRR stresses is the application of B. subtilis 10-4 in composition with SA, since this combination had a maximum positive effect on growth indicators (including growth in the lower (roots) and above-ground (shoots) tissues, as well as the accumulation of fresh and dry biomasses) of wheat, both under normal and drought stress conditions.

The obtained data on the effect of the studied B. subtilis 10-4, alone and in composition with SA, on the development of symptoms of FRR caused by F. culmorum and the intensity of plant growth processes under normal (non-drought) and combined drought stress conditions is in favor of the most effective use of the B. subtilis 10-4 + SA composition, both in stimulating growth and protecting plants from the damaging effects of combined drought + FRR stresses. The findings allow for the supposition that such physiological actions of B. subtilis 10-4 alone and in composition with SA may be connected with several possible mechanisms that require further thorough investigations to reveal the potential of these bioagents as a part of organic agriculture to improve wheat resistance/tolerance and productivity.

The earliest responses of plants to the impact of different stresses, including drought and infection with phytopathogens, is an oxidative explosion, i.e., the formation of reactive oxygen species (ROS) such as $\mathrm{H}_{2} \mathrm{O}_{2}$ and superoxide radicals, which harm the integrity of the membrane structures of plant cells $[1,34]$. As a result, oxidative damage in plant defense reactions are induced, including the synthesis of a diverse range of protective compounds $[2,34,35]$. Depending on the degree of susceptibility of the plant and the aggressiveness of the stress factor, these changes ultimately lead either to the formation of resistance or the development of disease and even plant death. Under oxidative stress, denaturation of proteins occurs in plant cells and large amounts of the products of lipid peroxidation accumulate, which can act as primary mediators of stress impact and inducers of plant defense mechanisms [34]. Thus, the development of plant protective reactions against stresses can be judged by the degree of malondialdehyde (MDA) concentration as the final products of lipid peroxidation. MDA content in leaves, representing degree of damage to the cell membrane, is commonly used to assess plant resistance/tolerance to stresses [36]. In our work, the analysis of the content of MDA in wheat seedlings using a color reaction with thiobarbituric acid allowed us to reveal the fact that artificial infection with F. culmorum resulted in increasing in MDA concentration in wheat plants, both under normal and combined drought conditions (Figure 7). These data indicate the intensive development of the pathogen F. culmorum in plant tissues and a decrease in their resistance/tolerance to pathogens and drought. Simultaneously, pre-sowing treatment with $B$. subtilis $10-4$, both alone and in composition with SA, facilitated a decrease in the Fusarium-instigated increase in MDA content, both under normal and combined drought stress (Figure 7), which indicates the induction of the protective reactions against drought, F. culmorum, and the combination of these stresses in plants. It should be noted that in healthy (non-infected) plants grown under normal conditions and pre-treated with B. subtilis 10-4, we observed a slight increase in MDA content also. Most likely, B. subtilis 10-4 can contribute (without 
harming the plant) to the preliminary adaptation of plants to the probable subsequent effects of stresses. With that, a change in the pro-antioxidant status of cells is the result of the action of only one of the links in a complex chain of biochemical and physiological metabolic processes.

Drought, FRR, and their combination are damaging factors that reduce plant growth and productivity, not only through the oxidative explosion but also due to water regime disturbance $[1,2]$. An important role in maintaining water status and preventing stress-induced damage in plants is played by the expression of genes coding for proteins with functions of molecular chaperones, proteases, and inhibitors of proteases. Moreover, under stressful conditions, plants synthesize low-molecular organic compounds such as sugars, betaines, and amino acids with osmoprotective properties [35], in particular, as a general metabolic adaptation allowing plants to cope with water or osmotic stress, including enhanced synthesis of substances such as proline (Pro). Pro is a multifunctional plant stress metabolite that plays an important role as an osmolyte, antioxidant, and low molecular weight chaperone, being involved in reducing the degree of stress-induced cellular structure damage [34, 35,37]. Thus, Pro is commonly used as an important biomarker to assess the formation of plant resistance/tolerance. Numerous data have shown that Pro increases in plants in response to a wide range of stresses and is important for survival of plants under extreme situations $[11,35,38,39]$. In the literature, there is evidence of the involvement of Pro in the protective activity spectrum of beneficial microorganisms, including Bacillus, in different plant species [1,9,39]. For instance, the use of beneficial bacteria increased biomass and the relative water content in maize leaves under drought stress, which was accompanied with increased Pro accumulation [1,40]. However, data on Pro changes in wheat plants under infection with Fusarium individually and in combination with drought stress, as well as the application of endophytic $B$. subtilis alone and particularly together with SA, have not been addressed before, and there was no information on the topic at the beginning of our work.

According to our findings, the content of Pro in wheat seedlings was markedly increased under drought and combined drought + FRR stresses in comparison to the control (Figure 8). In healthy plants under normal growth conditions, SA and B. subtilis 10-4 + SA also had a slightly increased Pro content (Figure 8). It is likely that a small activation of Pro accumulation caused by B. subtilis 10-4 can normally contribute to the strengthening of cell walls and subsequently provide effective neutralization of stress-induced ROS production, prevention of damage of cell membrane structures, and changes in the permeability during phytopathogenic attack and drought, since it is known that Pro acts as a mediator in the protective reactions of plants aimed at reducing the degree of the damaging effect of ROS on the integrity of the cell membrane structures $[37,38]$. It is most likely that B. subtilis 10-4-induced changes in Pro concentration, along with the role in osmoregulation, can also protect the structure of various biomolecules and membranes [37] and act as scavengers of free radicals, thereby protecting DNA against the damaging action of ROS [41].

\section{Conclusions}

In conclusion, this study provides an overview and novel insights into the interaction between endophytic B. subtilis, wheat, drought, and F. culmorum. The results presented here first establish that the pre-sowing treatment of wheat with endophytic bacteria B. subtilis 10-4 (capable of colonizing the internal tissues of wheat plants as well as producing IAA, siderophores, and fixed nitrogen), SA, and composition of B. subtilis 10-4 + SA significantly reduced FRR incidence in wheat seedlings and improved plant growth under normal, drought, FRR, and combined drought + FRR stresses, with the most pronounced positive effect being after application of $B$. subtilis 10-4 + SA. The obtained results suggest that the protective effect of tested endophytic bacteria B. subtilis 10-4 and SA on wheat plants under drought, FRR, and combined drought + FRR decrease upon their influence the degree of stress(es)-induced oxidative (MDA) and osmotic stresses (Pro) in plants. Thus, the studied B. subtilis 10-4 alone and especially in composition with SA has the potential to be used as an environmentally friendly agent to improve wheat growth and tolerance under drought, FRR, and combined drought + FRR stresses. 
Author Contributions: Conceptualization and methodology, O.L.; software and validation, O.L. and D.G.; formal analysis, D.G., O.L., I.K., A.V., C.A., and K.F.; investigation, O.L.; writing-original draft preparation, O.L.; writing-review and editing, C.A. and O.L.; visualization, O.L. and D.G.; supervision, project administration, and funding acquisition, O.L. All authors have read and agreed to the published version of the manuscript.

Funding: This research was funded by the Grant of President of the Russian Federation, grant no. MK-643.2019.11 and partially in accordance with the State assignment of Russia, no. AAAA-A16-116020350029-1.

Conflicts of Interest: The authors declare no conflict of interest.

\section{References}

1. Lastochkina, O.; Aliniaeifard, S.; Kalhor, M.S.; Yuldashev, R.; Pusenkova, L.; Garipova, S. Plant growth promoting bacteria-Biotic strategy to cope with abiotic stresses in wheat. In Wheat Production in Changing Environments: Management, Adaptation and Tolerance; Hasanuzzaman, M., Nahar, K., Hossain, A., Eds.; Springer: Singapore, 2019; Chapter 23; pp. 579-614. Available online: https://link.springer.com/chapter/10. 1007/978-981-13-6883-7_23 (accessed on 4 July 2020).

2. Pandey, P.; Ramegowda, V.; Senthil-Kumar, M. Shared and unique responses of plants to multiple individual stresses and stress combinations: Physiological and molecular mechanisms. Front. Plant Sci. 2015, 6, 723. [CrossRef] [PubMed]

3. Scherm, B.; Balmas, V.; Spanu, F.; Pani, G.; Delogu, G.; Pasquali, M.; Migheli, Q. Fusarium culmorum: Causal agent of foot and root rot and head blight on wheat. Mol. Plant Pathol. 2013, 14, 323-341. [CrossRef] [PubMed]

4. Moya-Elizondo, E.A. Fusarium crown rot disease: Biology, interactions, management and function as a possible sensor of global climate change. Cienc. Investig. Agrar. 2013, 40, 235-252. [CrossRef]

5. Peresipkin, V.F. Agricultural Phytopathology, 4th ed.; Agropromizdat: Moscow, Russian, 1989; 480p.

6. Liu, X.; Liu, C. Effects of drought-stress on Fusarium crown rot development in barley. PLoS ONE 2016, 11, e0167304. [CrossRef] [PubMed]

7. Sinha, R.; Irulappan, V.; Mohan-Raju, B.; Suganthi, A.; Senthil-Kumar, M. Impact of drought stress on simultaneously occurring pathogen infection in field-grown chickpea. Sci. Rep. 2019, 9, 5577. [CrossRef]

8. Egamberdieva, D.; Wirth, S.J.; Shurigin, V.V.; Hashem, A.; Abd_Allah, E.F. Endophytic bacteria improve plant growth, symbiotic performance of chickpea (Cicer arietinum L.) and induce suppression of root rot caused by Fusarium solani under salt stress. Front. Microbiol. 2017, 8, 1887. [CrossRef]

9. Lastochkina, O. Bacillus subtilis-mediated abiotic stress tolerance in plants. In Bacilli and Agrobiotechnology: Phytostimulation and Biocontrol; Islam, M.T., Rahman, M.M., Pandey, P., Boehme, M.H., Haesaert, G., Eds.; Springer Nature: Bremgarten, Switzerland, 2019; Volume 2, Chapter 6; pp. 97-133. [CrossRef]

10. Lastochkina, O.; Seifi Kalhor, M.; Aliniaeifard, S.; Baymiev, A.; Pusenkova, L.; Garipova, S.; Kulabuhova, D.; Maksimov, I. Bacillus spp: Efficient biotic strategy to control postharvest diseases of fruits and vegetables. Plants 2019, 8, 97.

11. Shakirova, F.M.; Avalbaev, A.M.; Bezrukova, M.V.; Fatkhutdinova, R.A.; Maslennikova, D.R.; Yuldashev, R.A.; Allagulova, C.R.; Lastochkina, O.V. Hormonal intermediates in the protective action of exogenous phytohormones in wheat plants under salinity: A case study on wheat. In Phytohormones and Abiotic Stress Tolerance in Plants; Khan, N., Nazar, R., Iqbal, N., Anjum, N., Eds.; Springer: Berlin, Germany, 2012; pp. 185-228.

12. Verma, P.; Yadav, A.N.; Khannam, K.S.; Kumar, S.; Saxena, A.K.; Suman, A. Molecular diversity and multifarious plant growth promoting attributes of Bacilli associated with wheat (Triticum aestivum L.) rhizosphere from six diverse agro-ecological zones of India. J. Basic Microbiol. 2016, 56, 44-58. [CrossRef]

13. Lastochkina, O.; Pusenkova, L.; Yuldashev, R.; Babaev, M.; Garipova, S.; Blagova, D.; Khairullin, R.; Aliniaeifard, S. Effects of Bacillus subtilis on some physiological and biochemical parameters of Triticum aestivum L.(wheat) under salinity. Plant Physiol. Biochem. 2017, 121, 80-88. [CrossRef]

14. Netrusov, A.I.; Egorova, M.A.; Zakharchuk, L.M. Praktikum Po Mikrobiologii (A Practical Course in Microbiology); Tsentr "Akademiya": Moscow, Russia, 2005.

15. Malik, D.K.; Sindhu, S.S. Production of indole acetic acid by Pseudomonas sp.: Effect of coinoculation with Mesorhizobium sp. Cicer on nodulation and plant growth of chickpea (Cicer arietinum). Physiol. Mol. Biol. Plants 2011, 17, 25-32. [CrossRef] 
16. Schwyn, B.; Neilands, J.B. Universal chemical assay for the detection and determination of siderophores. Anal. Biochem. 1987, 160, 47-56. [CrossRef]

17. Pikovskaya, R.I. Mobilization of phosphorus in soil in connection with vital activity of some microbial species. Microbiologiya 1948, 17, 362-370.

18. Umarov, M.M. Associative Nitrogen Fixation; Moscow State University: Moscow, Russia, 1986; 136p.

19. Mokronosov, A.T. Small Workshop on Plant Physiology; Moscow State University: Moscow, Russia, 1994; 184p.

20. Lastochkina, O.; Baymiev, A.; Shayahmetova, A.; Garshina, D.; Koryakov, I.; Shpirnaya, I.; Pusenkova, L.; Mardanshin, I.; Kasnak, C.; Palamutoglu, R. Effects of endophytic Bacillus subtilis and salicylic acid on postharvest diseases (Phytophthora infestans, Fusarium oxysporum) development in stored potato tubers. Plants 2020, 9, 76. [CrossRef] [PubMed]

21. Heath, R.L.; Packer, L. Photoperoxidation in isolated chloroplasts. Kinetics and stoichiometry of fatty acid peroxidation. Arch. Biochem. Biophys. 1968, 125, 189-198. [CrossRef]

22. Bates, L.S.; Waldern, R.P.; Teare, D. Rapid determination of free proline for water stress studies. Plant Soil 1973, 39, 205-207. [CrossRef]

23. Lastochkina, O.; Pusenkova, L.; Garshina, D.; Yuldashev, R.; Shpirnaya, I.; Kasnak, C.; Palamutoglu, R.; Mardanshin, I.; Garipova, S.; Sobhani, M.; et al. The effect of endophytic bacteria Bacillus subtilis and salicylic acid on some resistance and quality traits of stored Solanum tuberosum L. tubers infected with fusarium dry rot. Plants 2020, 9, 738. [CrossRef]

24. Rejeb, I.B.; Pastor, V.; Mauch-Mani, B. Plant responses to simultaneous biotic and abiotic stress: Molecular mechanisms. Plants 2014, 3, 458-475. [CrossRef]

25. Luo, M.; Liang, X.Q.; Dang, P.; Holbrook, C.C.; Bausher, M.G.; Lee, R.D.; Guo, B.Z. Microarray-based screening of differentially expressed genes in peanut in response to Aspergillus parasiticus infection and drought stress. Plant Sci. 2005, 169, 695-703. [CrossRef]

26. Kiraly, L.; Hafez, Y.M.; Fodor, J.; Kiraly, Z. Suppression of tobacco mosaic virus-induced hypersensitive-type necrotization in tobacco at high temperature is associated with downregulation of NADPH oxidase and superoxide and stimulation of dehydroascorbate reductase. J. Gen. Virol. 2008, 89, 799-808. [CrossRef]

27. Desprez-Loustau, M.-L.; Marçais, B.; Nageleisen, L.-M.; Piou, D.; Vannini, A. Interactive effects of drought and pathogens in forest trees. Ann. For. Sci. 2006, 63, 597-612. [CrossRef]

28. Chojak-Koźniewska, J.; Kuźniak, E.; Zimny, J. The effects of combined abiotic and pathogen stress in plants: Insights from salinity and Pseudomonas syringae pv lachrymans interaction in cucumber. Front. Plant Sci. 2018, 9, 1691. [CrossRef] [PubMed]

29. Navas-Cortés, J.A.; Hau, B.; Jiménez-Díaz, R.M. Yield loss in chickpeas in relation to development of Fusarium wilt epidemics. Phytopathology 2000, 90, 1269-1278. [CrossRef]

30. Tarafdar, A.; Rani, T.S.; Chandran, U.S.S.; Ghosh, R.; Chobe, D.R.; Sharma, M. Exploring combined effect of abiotic (soil moisture) and biotic (Sclerotium rolfsii Sacc.) stress on collar rot development in chickpea. Front. Plant Sci. 2018, 9, 1154. [CrossRef]

31. Gaur, P.M.; Jukanti, A.K.; Samineni, S.; Chaturvedi, S.K.; Basu, P.S.; Babbar, A.; Jayalakshmi, V.; Nayyar, H.; Devasirvatham, V.; Mallikarjuna, N.; et al. Climate change and heat stress tolerance in chickpea. In Climate Change and Plant Abiotic Stress Tolerance; Tuteja, N., Gill, S.S., Eds.; Wiley-VCH: Berlin, Germany, 2013; Chapter 31; pp. 241-246.

32. Achuo, E.A.; Prinsen, E.; Hofle, M. Influence of drought; salt stress and abscisic acid on the resistance of tomato to Botrytis cinerea and Oidium neolycopersici. Plant Pathol. 2006, 55, 178-186. [CrossRef]

33. Bowler, C.; Fluhr, R. The role of calcium and activated oxygens as signals for controlling cross-tolerance. Trends Plant Sci. 2000, 5, 241-246. [CrossRef]

34. Kolupaev, Y.E.; Yastreb, T.O. Physiological functions of nonenzymatic antioxidants of plants. Proc. KhNU. 2015, 2, 6-25.

35. Szabados, L.; Savoure, A. Proline: A multifunctional amino acid. Trends Plant Sci. 2009, 15, 89-97. [CrossRef]

36. Del, R.D.; Stewart, A.J.; Pellegrini, N. A review of recent studies on malondialdehyde as toxic molecule and biological marker of oxidative stress. Nutr. Metab. Cardiovasc. Dis. 2005, 15, 316-328. [CrossRef]

37. Hare, P.D.; Cress, W.A.; Van Staden, J. Dissecting the roles of osmolyte accumulation during stress. Plant Cell Environ. 1998, 21, 535-553. [CrossRef] 
38. Molinari, H.B.C.; Marur, C.J.; Daros, E.; de Campos, M.K.F.; de Carvalho, J.F.R.P.; Filho, B.J.C.; Pereira, L.F.P.; Vieira, L.G.E. Evaluation of the stress-inducible production of proline in transgenic sugarcane (Saccharum spp.): Osmotic adjustment, chlorophyll fluorescence and oxidative stress. Physiol. Plan. 2007, 130, 218-229. [CrossRef]

39. Allagulova, C.R.; Lastochkina, O.V. Alleviation of drought-induced oxidative stress in wheat plants under the influence of endophytic bacteria-Review. Ecobiotechnology 2020, 3, 129-134.

40. Sandhya, V.; Ali, S.Z.; Grover, M.; Reddy, G.; Venkateswaralu, B. Effect of plant growth-promoting Pseudomonas spp. on compatible solutes antioxidant status and plant growth of maize under drought stress. Plant Growth Regul. 2010, 62, 21-30. [CrossRef]

41. Ashraf, M.; Foolad, M.R. Roles of glycine betaine and proline in improving plant abiotic stress resistance. Env. Exp. Bot. 2007, 59, 206-216. [CrossRef]

(C) 2020 by the authors. Licensee MDPI, Basel, Switzerland. This article is an open access article distributed under the terms and conditions of the Creative Commons Attribution (CC BY) license (http://creativecommons.org/licenses/by/4.0/). 